Décadrages Décadrages

cinéma, à travers champs Cinéma, à travers champs

$7 \mid 2006$

Stephen Dwoskin

\title{
Entretien avec Stephen Dwoskin : filmographie commentée par l'auteur
}

\section{Stephen Dwoskin et François Bovier}

Traducteur : François Bovier

\section{OpenEdition \\ Journals}

\section{Édition électronique}

URL : https://journals.openedition.org/decadrages/447

DOI : $10.4000 /$ decadrages. 447

ISSN : 2297-5977

\section{Éditeur}

Association Décadrages

\section{Édition imprimée}

Date de publication : 10 avril 2006

Pagination : 63-88

ISBN : 978-29700582-3-6

ISSN : 2235-7823

\section{Référence électronique}

Stephen Dwoskin et François Bovier, « Entretien avec Stephen Dwoskin : filmographie commentée par l'auteur », Décadrages [En ligne], 7 | 2006, mis en ligne le 30 janvier 2014, consulté le 12 avril 2022.

URL : http://journals.openedition.org/decadrages/447 ; DOI : https://doi.org/10.4000/decadrages.447 


\section{Entretien avec Stephen Dwoskin : filmographie}

\section{commentée par l'auteur}

Correspondance électronique avec François Bovier, du 3 novembre 2005 au 21 janvier 2006

François Bovier - Est-il légitime de départager ta pratique filmique en différentes périodes, ce qui permet d'ordonner chronologiquement le corpus suivant deux ou trois axes principaux? Stephen Dwoskin - Au sujet du découpage de mon travail en "différentes périodes»: je pense que ton résumé est juste dans les grandes lignes, même si la première et la seconde "périodes» sont plus liées que tu ne le suggères. Pendant la première période, je suis la caméra (et la caméra en tant qu'acteur); pendant la seconde, je deviens la caméra à la fois invisible et vue. Le flux et le point de vue, toutefois, ne changent pas - seule une légère (mais importante) inflexion intervient...

FB - II y a peut-être un sens à appréhender tes pr

SD - Je dirais volontiers que mes premiers films répondent, en premier lieu, à un processus d'exploration. Asleep ou American Dream, par exemple, constituent avant tout des expérimentations sur le maniement de la caméra et la dimension temporelle. Je partais d'une pratique plus statique (la peinture, la photographie et le graphisme). Il était nécessaire, en un premier temps, d'élargir mon vocabulaire, de m'approprier l'« œil» de la caméra comme mon «œil». Des films comme - Alone sont à l'origine de ma longue relation avec le sujet filmé comme, à la fois, celui qui est vu, celui qui retourne son regard et qui voit. La relation avec mes «modèles» (pour reprendre ce terme) ne répond pas à des fondements théoriques. Les films reposent assez manifestement sur une "relation" à l'autre et réfléchissent dans le même temps cette relation, puisque les «modèles» nouent en retour un rapport à ma personne. (Il s'agit d'un dialogue, en quelque sorte.) En un sens aussi (et de façon diversifiée) mes films occupent indissociablement la position de témoin et de participant à cette relation. J'avais l'intention d'explorer la mécanique de ces relations denses à travers un certain laps de temps, tout comme on suit une pensée, comme on passe un moment avec quelqu'un (ou Approximativement, nous pourrions soutenir qu'il y a une première période, lorsque tu filmes des modèles (féminins), et une seconde, lorsque tu retournes la caméra contre toi. Entre-deux, il y a des expérimentations sur des adaptations littéraires et une alternative à la narration traditionnelle. Après la première phase et jusqu'à ce jour, il y a une série de films documentaires. Que penses-tu de ce découpage?

premiers courts métrages comme un ensemble cohérent, en termes de procédés filmiques aussi bien que de conditions de tournage. - Asleep (1961), - American Dream (1961), - Alone (1963), - Naissant (1964), - Chinese Checkers (1963/1965), Dirty (1965-1971), - Soliloquy (1964/1967), - Take Me (1968), - Me, Myself and I (1967), - Moment (19681969) et $>$ Trixi (1969) reposent tous sur un face à face entre l'homme à la caméra et des modèles féminins (dans le sens où Kouléchov l'entendait: un modèle, et non pas un acteur). Pourtant, différents modes de relations sont impliqués, les interactions étant tout aussi bien binaires que triangulaires.

Sur un plan général, comment conçois-tu ton approche qui met en jeu une dynamique intersubjective des regards, théorisée par Paul Willemen comme un quatrième regard? Peut-on relier ou au contraire différencier certains films suivant la relation que tu entretiens avec les modèles et les consignes que tu leur donnes avant de tourner? 
avec soi-même). Les films doivent ressaisir l'impression laissée par ces moments singuliers (ou ces instants) plutôt que constituer une narration en tant que telle. Pendant le tournage, la conclusion (des films) n'est jamais prévisible.

Pourquoi porter son regard sur des femmes, me demande-t-on toujours? Je pense que le «dialogue» que j’ai filmé repose sur le «désir» que j'éprouve envers mes interlocuteurs. Tous les films de cette période présentent un potentiel élevé (plus ou moins intense) d'implications sexuelles. Il ne s'agissait pas pour moi d'entretenir une relation voyeuriste avec le «modèle» mais de susciter une participation "réelle» (c'est un point important) - il s'agit d'être impliqué -, le mode de participation de la caméra et la reconnaissance par le «modèle» de sa présence (et de la personne qui se tient derrière la caméra) en dépendent. Cette reconnaissance représente le principal point de pivot entre les positions de voyeur et de participant.

Dans - Alone ou $\bullet$ Moment, le regard est souvent assimilé à un coup d'œil; dans $\triangleright$ Trixi ou $\bullet$ Take $M e$, il s'agit presque tout le temps d'un regard fixe et continu. Dans un film comme $\bullet$ Me, Myself and I, la présence de la caméra est soulignée par la conscience de son évitement. Les «thèmes» de tous ces films portent sur des relations (avec l'autre, avec soi; avec le moi, la caméra et l'autre - qui devient le spectateur/regardeur!) (Je pense que ce sont ces interactions qui mettent en jeu une «dynamique des regards» que Paul Willemen (et Laura Mulvey) ont théorisé comme un quatrième regard!) Je dois ajouter que ces films participent à une «exploration" plus vaste qui porte sur ma personne et le cinéma - pour m’approprier un langage cinématographique personnel - qui peut être élargi et utilisé dans d'autres contextes, avec d'autres thèmes.

Je dois encore préciser que ces premiers films participent de façon critique à une préoccupation personnelle : comment «négocier un accès" au moi - à l'espace intérieur du moi - tel qu'il se déploie mentalement lorsque nous sommes seuls. J'étais très influencé par les écrits de James Joyce (tout particulièrement Ulysse) et par certains passages comme le soliloque de Molly Bloom (mon film $>$ Soliloquy, en est directement inspiré). Je cherchais le moyen de tranposer le sens des mots en images - de parler avec les yeux, en quelque sorte - et tout particulièrement de transposer les processus internes de la pensée en une représentation visuelle.

FB - Parmi tes premiers courts métrages, tu opères une distinction entre deux ou trois types

de regards. En premier lieu, tu as parlé de "coup d'œil " par rapport à $>$ Alone et $>$ Moment. En second lieu, tu as parlé de "regard fixe" par

SD - En ce qui concerne - Moment et - Alone - oui, il y a différents types de "cadrages", mais leur différence est relative. Tous deux sont pris 
depuis une position fixe: $\bullet$ Alone utilise différents cadrages (en gros plan ou plan semi-large) mais depuis une position fixe de caméra (depuis le pied du lit), alors que - Moment adopte une position de caméra absolument fixe (au-dessus de la femme). Je m'oppose pour une part à ta remarque: "Dans - Alone, il y a une multiplicité de points de vue, une réelle discontinuité» - Alone représente une continuité depuis un point de vue unique malgré l'effet cumulatif de durée dû au montage, alors que $\rightarrow$ Moment repose sur une prise unique assimilant le film au temps «réel» (je suppose qu'il s'agit là de deux structures différentes).

Suivant comment tu définis le "voyeurisme», - Alone est plus voyeuriste que -Moment: la caméra est le principal agent du "coup d'œil» dans $\bullet$ Alone, alors que dans $\bullet$ Moment la femme (le modèle) reconnaît consciemment la présence de la caméra (et celle du spectateur). Tu suggères que dans •Moment la "masturbation" est située dans un autre mode de représentation (parce qu'on ne voit pas l'ensemble du corps de la femme, je suppose). Pour moi, il s'agit avec ce film de "faire corps» (d'être en relation) avec l'expression de l'«orgasme» - d'être témoin (de partager ce moment), d'accompagner ce sentiment intime d'être en communion «avec soi»! ( Alone et $\vee$ Moment présentent tous deux cette similitude - d'être avec (le modèle) dans des situations d'intimité.)

Je pense que la relation à certaines œuvres de Warhol est avant tout incidente: la fascination pour la caméra «motorisée» et les structures en «temps réel» (comme dans Wavelenght, etc.) répondait à une volonté d'élargir les instruments alors disponibles (c'est probablement le point de vue retenu par l'Histoire - si la vidéo était alors accessible, qui sait ce qui se serait pratiqué!)- et cette fascination doit être rapportée à la notion de temps «ininterrompu» (non monté, non manipulé). Sleep de Warhol, par exemple, reposait sur six boucles montées, alors qu'Empire était constitué de prises continues. ( Alone aurait pris une tout autre forme si j'avais disposé d'une caméra motorisée plutôt qu'à ressort, limitant les prises de vue à une durée de 26 secondes...)

En un sens, le fait que Trixi soit danseuse est accidentel. La relation porte plutôt sur la mobilité (qui me fait défaut et dont elle abonde - le film porte sur sa fascination pour mon absence de mobilité et sur mon attirance pour son abondance de mouvement - ou encore sur la mobilité de mon œil faisant face à la mobilité de son corps!) Il faut évidemment prendre ici en considération mon handicap, et ses effets sur ma relation aux autres - m'affixant à un singulier point de vue «fixe». Quand j'ai commencé à faire des films, je ne pensais pas vraiment à cela... Aujourd'hui, avec le recul, je me rends compte que cet aspect était décisif dans mon exploration d'un "point de vue» et de différents types de regards... rapport à $\$ Trixi et $\$ Take Me. En troisième lieu, tu as parlé d'un "évitement de la conscience de la caméra" par rapport à $>$ Me, Myself and $I$.

Dans le premier cas, tu te concentres sur une fille dont la position varie lentement avec le temps. Dans $>$ Alone, il y a une multiplicité de points de vue, une réelle discontinuité qui souligne l'attention flottante du modèle. Par contre, dans $>$ Moment, tu optes pour un plan fixe, et tu rejettes de surcroît hors-champ ce qui peut être considéré dans un autre mode de représentation comme le clou du film (la masturbation). II me semble que tu recours là à deux types radicalement différents de cadrages. Je ne peux pas m'empêcher de penser, face à - Moment, à un déplacement du regard voyeuriste fixe de Warhol, que tu mets radicalement en question (ici sur un mode implicite, par la suite ouvertement). Quel est ton point de vue sur ce sujet?

Dans le second cas, nous entrons franchement, du moins du point de vue du public, dans une dynamique intersubjective entre l'opérateur et le modèle. Pour le dire franchement, je doute que l'on puisse encore parler de regard fixe. Peux-tu préciser sur ce point ta relation, qui me semble cruciale, à la danse et à la chorégraphie - qui est de fait liée à $>$ Trixi? 
FB - La notion d'interaction dialogique, intersubjective, est centrale pour appréhender ton $œ u v r e$. Je dirais volontiers que tu postules une différenciation qui porte sur deux niveaux.

D'une part, il y a une expérimentation sur le support filmique et sa dimension temporelle: comment cadrer un sujet, maintenir l'intensité de la durée qui s'écoule; comment évoluer d'une image statique à une mise en relation de plans mouvants.

D'autre part, tu fais allusion à une sphère de relation privée: il y a le sujet ausculté qui fait face à ton regard et qui dévisage la caméra, et par là-même le public. Cette relation fissure le mode de représentation hollywoodien: en lieu et place d'un regard voyeuriste, tu instaures une interaction entre les instances filmique et profilmique, reposant sur une large part d'improvisation pendant le tournage.

$Y$ a-t-il une relation intrinsèque entre la recherche d'un cadrage ajusté du sujet féminin et l'exposition à une expérience intersubjective que tu partages avec le modèle? Enfin, la relation entre ton immobilité et la relative mobilité du sujet filmé intervient-elle sur ce point?

SD - Une idée me vient à l'esprit - je transpose (à travers le film; la caméra; le montage) ce que j’ai et ce que je ressens en ce dont je suis privé et que je désire. C'est la base du dialogue!

Le «cadrage» (et la poursuite du cadre) institue le «regard»- le visage dévisageant, et le «visage» dirigeant les mouvements corporels par les yeux (de près comme de loin). Les yeux apparaissent comme une extension de l'«esprit» - comme l'expression d'une intime subjectivité qui se manifeste à l'extérieur - à travers l'expression du visage - et le «regard fixe» des yeux / par les yeux. La caméra est une extension de l'œil - qui regarde et capture spontanément la scène en se rapportant, en répondant, en réagissant à l'autre (à la réponse, à l'état d'esprit, au sentiment du modèle)...

Cela forme mon "histoire», pour ainsi dire. La situation environnante (ou l'environnement de l'action) constitue une simple toile de fond pour la poursuite de ce regard - dans ce but, dans cette intention. Pour moi, il s'agit de trouver un moyen d'exprimer comment une personne (y compris moi-même) se perçoit intérieurement - le film doit projeter à l'extérieur, comme une réflection (un miroir), ce sentiment, ou cette série de sentiments, cette constante métamorphose d'un sentiment en un autre. (Le cinéma constitue un essaim complexe de sensations, de peurs, de désirs, etc. Le cinéma - grâce à sa durée et à son processus de confrontation - constitue une tentative d'extraction - de mise en jeu - de présentation - de questionnement - de traduction de ce réseau interne à travers le potentiel réflexif de l'image en mouvement.)

Quant à la question de la mobilité: il s'agit d'une limitation sur le plan physique, mais l'esprit, l'œil et le regard pallient les limitations physiques à travers leur mobilité intérieure; à travers leur subjectivité - qui met en jeu un mode de mobilité "mentale». Je suppose que je substitue à la physicalité manifeste un autre mode de physicalité.

FB - Dans D Dirty, tu établis une relation entre des femmes nues et un grain de pellicule très

matériel. Y a-t-il un rapport affirmé, conscient, à la peinture expressionniste?

SD - Dirty explore tout simplement le matériau filmique (le re-filmage découvrant des rayures, des ratures, des sautes entre photogrammes), ainsi que le jeu "analytique» des mouvements et des gestes des filles, de leurs actions, à travers le procédé du re-filmage. Il est question de la nature du film, si tu veux. L'un des résultats de ce procédé revient à attribuer aux images un caractère plus "éclatant", plus "granuleux", plus «graphique» ou plus «photographique» - je suppose que l'on peut considérer cela comme expressionniste. 
FB - Comment es-tu passé d'une forme relativement brève aux longs métrages? Quelles sont

SD - D'après moi, - Times for et $\boldsymbol{\bullet}$ DynAmo sont très proches sur un plan conceptuel, même si l'espace ou la sphère où ils se déroulent sont différents. Tous deux partagent la même thématique - la femme instrumentalisée par les désirs sexuels masculins - la femme comme objet de ce désir et éprouvant un déplaisir face à sa propre exhibition (cette contradiction est au centre de la stéréotypie entre sujets masculin / féminin) - ou : "nous sommes l'image à laquelle on nous demande de nous conformer - et que nous haïssons». Dans — Times for, les femmes apparaissent «là» en tant que "types» que le mâle rencontre (quatre fois). (Il s'agit d'une référence à "Night-Town»- l'homme [Bloom] au bordel.) •DynAmo incarne la théatrâlité du Strip-tease. La Mise à nu de la femme s'adresse au spectateur masculin. Les deux films sont apparentés: mon intérêt ne porte pas sur l'acte sexuel ou sur le strip-tease, mais sur les femmes et l'expression de leurs «sentiments» par rapport à leurs comportements et contradictions - par rapport à leur position (se heurtant aux stéréotypes et aux symboles de l'exploitation) - pour revenir sur leurs visages et leur(s) «regard(s)». (D’après moi, il faut regarder par-delà l'évidence - le stéréotype - pour "démasquer» le "masque» que les gens revêtent - si l'on me passe l'expression.)

Oui, - Times for correspond (au sein de mon corpus) à la transition de films courts à des films plus longs. Pour quelle raison? - Parce que mes idées sont devenues plus vastes et complexes - elles exigent plus de temps. Ces films participent à un processus d'autoexploration. Dans - Times for, on assiste à une transition encore maladroite, puisque le film peut être décrit comme un assemblage de quatre courts métrages; dans - DynAmo, la fusion en un film unique est plus accomplie (le film est également découpé en quatre parties).

FB - Dans ces deux films, ton travail consiste à

SD - J'élabore souvent une image stéréotypée (une action, un geste) qui met en place ce qui est "attendu" (par le spectateur) - i. e. le striptease est censé révéler les parties intimes du corps. Au lieu de poursuivre dans cette direction, je porte mon «regard»sur la «personnalité» et les «sentiments» de l'individu qui incarne le stéréotype (le plus souvent à travers ses expressions faciales), suspendant ainsi l'action "attendue " pour recadrer le «regard» sur l'individu et saisir sa relation individuelle à l'acte accompli. J'établis ainsi une nouvelle relation en distendant le temps consacré à regarder l'individu (contrecarrant ce qui est attendu) - le point de vue se déplace ainsi de façon inattendue, le regard (du les implications d'une durée plus étendue sur ton filmage et ton montage?

- Times for (1971) - ton passage au long métrage - et $>$ DynAmo (1972) répondent à une dynamique différente. Le premier est concentré sur la sphère privée (intime), le second se déroule sur une scène de théâtre. Peux-tu m'en dire plus à ce sujet? littéralement "mettre à nu" le sujet - à démasquer les masques (sociaux, culturels, etc.) ou, pour le dire autrement, à peler les différentes couches de peaux d'un oignon. De ton point de vue, sur quels moyens filmiques (le cadrage, mais aussi le montage) et sur quels modes de relations (avec les sujets) cette mise à nu repose-t-elle? 
spectateur - et aussi de la caméra) devant être ré-établi (en incluant les «modèles»). Sans doute, ce renversement d'un point de vue voyeuriste (construit par le décor stéréotypé) en une expression individuelle inattendue (impliquant toutes les parties - le spectateur, l'interprète, la caméra) conduit à une confrontation qui implique personnellement le spectateur, qui doit répondre à ce qu'il voit. Selon moi, ce processus n'est pas voyeuriste (autrement dit: regarder sans investissement personnel - «se laisser dicter ses réactions à travers un regard passif») mais implique une interaction entre le spectateur et l'image qui bouleverse la vision - de passive, celle-ci devient active. (Ce qui dépend directement du «montage dans la caméra» et non du découpage sur la «table de montage»!)

FB - DynAmo repose sur une pièce de Chris Wilkinson, jouée par le Soho Theatre. Peux-tu me dire quelques mots de cette pièce et de l'interprétation que tu en as faite (pendant la mise en scène, le tournage et le montage)?

SD - L'histoire de $\bullet$ DynAmo et de la pièce est simple, dans ses grandes lignes. Un producteur m'a demandé de tourner un film à partir de la pièce. La Pièce était jouée sur une scène de pub. J'ai dit que je ferais le film, mais quand j'ai vu la pièce, ce que ressentaient les inteprètes (en eux-mêmes) accomplissant le strip-tease m’a intéressé. (La pièce, à mon souvenir, portait sur le strip-tease, sur la scène et sur les femmes dans les coulisses - la scène était départagée en deux pour montrer cette scission.) Quand j'ai réalisé le film, j’ai poursuivi mes propres intérêts (ce dont nous avons déjà parlé en partie: i. e. le masque, le démasquage et les contradictions, etc.). Je l'ai tourné dans un ancien club de Strip de Londres (sans public) et non dans un environnement théâtral. Je n'ai à aucun moment été impliqué dans la Pièce; je n'ai pas lu, ni rencontré l'auteur, aussi je ne sais pas ce qu'il a pu en penser. Le producteur du film attendait probablement quelque chose de plus exploitable. Je ne l'ai pas vu après le tournage du film, et du reste je n'ai pas reçu un sou! Le reste appartient désormais à l'histoire.

FB - Une chose m'a frappé, en revoyant $>$ DynAmo. Le strip-tease, après un moment, bascule

dans une scène vraiment violente (un happening extrême, en quelque sorte). Et le dernier plan renvoie explicitement à une représentation de la crucifixion. En un sens, ainsi, - DynAmo parle autant du strip-tease que de la violation et du viol. (Tout au moins, c'était mon impression. Le long plan fixe sur la fille en pleurs vers la fin n'est pas étranger à mon impression.) Quelle était ta position, ton intention, en affrontant, en recaptant cette scène?

sD - J'avais l'intention, avec •DynAmo, de basculer d'une "performance» (un spectacle) à une forme violente de "réalité» opposant hommes et femmes (impliquant une confusion entre le «joué» et le "non joué»). Il s'agissait d'explorer le «jeu de pouvoir» sexuel et de situer (placer) les hommes (ou les mettre eux-mêmes) en relation avec des femmes qui se présentent en tant qu' "objets" sexuels (une forme d'icône sensuelle qui retient une part de son "pouvoir» initial); il s'agissait d'exposer com- 
ment les femmes (et tout un chacun, en ce cas) sont piégées (enfermées) par cette «icône» qui trahit leurs sentiments - l'apparence extérieure étant en flagrant désaccord avec ce que la personne ressent intérieurement. En un sens, il s'agissait de démontrer que le voyeurisme constitue un piège qui oblitère le sentiment de celui (celle) qui est vu(e)/par ceux qui voient. Le «long plan» de la «fille» (qui pleure) met à nu cette confrontation. Elle appelle à l'«aide»! Elle veut quitter la scène ! Le spectateur n'est plus un "voyeur» passif: il doit faire face à cette situation de détresse sans être d'aucune aide. Le tournage répond aussi à ce que tu as caractérisé comme une "violence» dans le film - au début, le spectateur est conscient de la présence de la "scène». Tandis que le film progresse, la caméra s'approche de plus en plus du spectacle, et la «scène» disparaît presque complètement. Les personnages sont vus en gros plans et sont constamment "étudiés" en dehors de la "scène» (il s'agit encore d'une forme de «mise à nu»). Ce processus, couplé au "plan long», retourne le film de l'«extérieur» à l'«intérieur». Le plan final revient à l'espace de la scène - où tu relèves la pose de "crucifixion". Je n'ai pas pensé littéralement à la "crucifixion" - il était question d'une pose de "soumission" ou d'abandon (mais la crucifixion implique la même idée). Il s'agit aussi d'une pose de cirque que les interprètes composent à la fin de la scène - à l'exception de la femme qui est nue, à l'opposé des hommes (encore une contradiction!). Ils apparaissent comme les dépositaires en souffrance du cirque sexuel qui se tient derrière le spectacle. (La bande son pastiche aussi la musique de «cirque».)

FB - Quelle est ta relation au minimalisme, tout particulièrement dans le domaine de la muSD - Ma relation à la musique (à mon sens) est très étrange (de mon point de vue). Je ne sais jouer d'aucun instrument, je ne peux pas lire une partition, je n'ai pas «l'oreille mélomane» et je ne peux même pas fredonner une chanson; par contre, j'aime toute sorte de musique, de façon tout à fait désordonnée. J’aime également les opéras et la musique country; le minimalisme et le romantisme, etc. La musique, c'est du son organisé, et j'aime le flux et l'espace que me procure la combinaison de sons variés. Ce sont souvent des "passages», et non des morceaux entiers de musique, qui me paraissent puissants et que je reproduis (mentalement). Ma préférence se porte sur de simples variations au sein de longs passages de musique - telles que Malher ou Ives les pratiquent; ou sur des répétitions périodiques telles que Steve Reich les compose (ou le chant Grégorien, en la matière). Je ne peux pas dire à quel point mes films sont redevables de ce modèle, mais j'ai commencé à comprendre sique? Le minimalisme entretient-il, de ton point de vue, une relation au cinéma structurel? Par ailleurs, es-tu influencé par un mode de composition musicale (impliquant un rythme régulier, un calcul de la durée des plans, voire du nombre de photogrammes)? 
le montage en écoutant de la musique. Autrement dit, la question porte sur «la mise en forme et la construction du temps». (La musique est une métaphore qui ne peut pas être transposée mécaniquement.) Il ne s'agit pas de cinéma "structurel» (comme type de pratique filmique), mais de structurer dans le temps les images, ce qui est à la base du cinéma. Pour répondre plus précisément à ta question : la musique "minimale» est peut-être théoriquement reliée au cinéma "structurel", mais pas dans la pratique. Le langage de l'image (en mouvement) implique une autre signification (une sensibilité différente) que celui du son, et s'ils peuvent s'allier (en un équilibre délicat) ils constituent néanmoins des formes d'expression distinctes et des modes d'expérience divergents. Dans presque tous mes fims, le son (ou la musique) a été composé après l'assemblage des images. En termes pratiques, nous pourrions donc soutenir que ce sont les images qui ont influencé le son!!!

FB - J'avais à l'esprit une question plus précise: calcules-tu la durée des plans et établis-tu des mesures d'ordre métrique entre eux, ou le rythme filmique dépend-il de la durée d'une simple prise de vue (je pense tout particulièrement à tes courts métrages des années 1960 et 1970)?

SD - La durée des plans ne dépend d'aucune mesure métrique (dans le sens mathématique ou mécanique du terme). La durée dépend des mouvements (gestuels ou expressifs) à l'intérieur du plan individuel (ou de la «prise»). Parfois (le plus souvent) la durée prend forme au moment du tournage, puis du montage. En fin de compte, il s'agit d'une combinaison de ces deux phases - d'une relation des plans entre eux en suivant le(s) mouvement(s) à l’intérieur des plans individuels. En règle générale, le «rythme» n'est pas préconçu - les «rythmes» s'agencent en fonction du matériau concret accumulé. Des éléments «rythmiques» (ou chorégraphiques) instinctifs interviennent certainement pendant le tournage, mais ils ne sont pas conscients!

FB - Si je ne me trompe pas, Laura Mulvey connaissait tes films avant d'écrire son fameux article sur "le plaisir visuel et le cinéma narratif". Peux-tu me dire comment elle réagissait face à tes films? Et s'ils ont eu, de ton point de vue, une influence sur sa détermination de trois regards au cinéma, principalement voyeuristes et fétichistes (nous pourrions dire que tes films exhibent et mettent en crise cette dynamique scopique)?

sD - Oui, Laura connaissait mes films avant d'écrire son essai «Visual Pleasure and Narrative Cinema». (En fait, nous étions amis et voisins à l'époque!) Elle disait que mes films étaient d'abord «difficiles» à supporter pour elle (d'un point de vue féministe), mais qu'ils ont par la suite largement contribué à l'élaboration de sa thèse énoncée dans «Visual Pleasure and Narrative Cinema». ( $\bullet y$ Dymo, $\triangleright$ Times for et $\bullet$ Behindert, 1974, étaient probablement ses principales références.) Ainsi, mes films ont probablement «influencé» sa formulation du «plaisir visuel et du cinéma narratif». 
FB - Après ton premier long métrage, tu reviens à la forme brève. Jesus' Blood (1972) constitue une brillante interaction entre un extrême

SD - Jesus' Blood repose sur une idée simple. Il s'agissait - d'accomparalenti et le chant monté en boucle d'un clogner le "vieil homme» aux cieux. En une lente avancée, le vieillard - comme s'il baignait à travers le grain du film - s'efforce de s'approchard. Comment conçois-tu l'interaction entre son et image dans ce cas? cher mais n'y parvient pas. Il est question du temps, de son ralentissement, de sa disparition, etc. Le chant provient d'un vieil enregistrement sonore réalisé pour un documentaire sur les alcooliques. Il a été mis en boucle et orchestré pour être joué avec le film dans une performance «en direct» (La première projection du film a eu lieu dans un théâtre avec le film projeté sur un écran large et la musique inteprétée par un orchestre en direct, avec la voix mise en boucle sur une cassette. La musique a été enregistrée et ajoutée au film après-coup.) En un mot - le son était supposé accomplir ce que réalisait l'image. (Intensifier à travers la répétition!)

FB - $>$ Tod und Teufel (1973), ton troisième long métrage, est une adaptation de Frank Wedekind. Comment as-tu retravaillé la pièce? Et quelle relation ton film entretient-il avec La Boîte de SD - Le texte original de $\neg$ Tod und Teufel a été modifié: certaines parties Pandore (G. W. Pabst)?

ont été ré-écrites et d'autres ajoutées au texte de Wedekind. Le texte original était daté (même s'il porte sur l'ambiguïté sociale des femmes - et sur les erreurs que les hommes commettent dans leur relation aux femmes). Carola Regnier (la petite-fille de Wedekind) et Charles Regnier (le père de Carola et le beau-fils de Wedekind) ont participé à la réécriture, et comme ils jouaient tous deux dans le film, j'ai eu un accès privilégié aux intentions de Wedekind. Une part importante du texte a été modifiée (mais pas le thème) en recourant au langage contemporain (des années 1970) du "féminisme» (avec ses contradictions!). De toute façon, comme Wedekind n'a pas vraiment achevé sa pièce, j’ai pris certaines «libertés » dans le film pour élargir son idée initiale - j’ai ainsi pris le parti de présenter le dialogue et les personnages sous un tout autre angle.

- Tod und Teufel devait constituer la troisième pièce du cycle de Loulou. Pabst, comme tu le sais, n'a utilisé que les deux premières Pièces ( L'Esprit de la terre et La Boîte de Pandore). Le principal lien à Pabst tient au fait que j'espérais vaguement pouvoir compléter le cycle avec - Tod und Teufel. J'admire sincèrement le travail de Pabst, mais ma seule relation (stylistique) à ses films a peut-être trait à la façon dont Louise Brooks interprétait Loulou. Mon rapport à la famille Wedekind et à des informations d'arrière-plan auxquelles j'ai eu accès (comme consulter le manuscrit original!) était plus important. Le thème de la pièce - les contradictions entre les rôles masculins / féminins et leur interrelation - constitue la pincipale influence de mon film. 
FB - La caméra commence par lentement s'approcher du visage du modèle, en gros plan.

Ensuite le film passe à un long dialogue, avec la caméra qui erre dans l'espace, entre les deux protagonistes. Comment as-tu conçu ce renversement au niveau de la représentation? Quel type de relation peut-on établir entre la première et la deuxième partie?
SD - La longue séquence de dialogue constitue, bien sûr, le centre du film. La séquence précédente (avec la jeune femme Lisiska) présente la «réalité» de la femme à laquelle l'homme va mentalement se référer comme à un «idéal» d'une "pure sensualité, non mélangée». C’est là son erreur. Et lorsqu'il rejette la femme avec qui il parle, il est à son tour rejeté : il comprend que la jeune femme n'est pas celle qu'il a imaginée ; et dans le récit, son ego autocentré est dépourvu de toute signification pour la seule femme libre (la dernière femme). Son argumentation est ainsi mise à bas, et sa personne mise à nu. (Dans le film, tout se passe en un seul jour, dans les différentes chambres d'une même maison.) (L'intention de Wedekind dans cette Pièce était d'exhiber ce qui arrive à Casta Piani [l'homme] lorsqu'il a réduit Loulou à la prostitution.)

FB - Tod und Teufel thématise les difficultés de communication et de relation entre la gente masculine et féminine. Peut-on dire que le film, par le biais de la pièce, constitue un processus réflexif qui porte sur ta propre pratique filmique?

sD - Tod und Teufel, pour une part, est le seul film à représenter thématiquement (sur un plan général) un procesus (une situation) extérieur plutôt qu'un processus réflexif - présent surtout dans mes premiers films. Aujourd'hui c'est encore moins le cas: mon attention porte avant tout (ou de surcroît) sur la voix intérieure réflexive (l'espace intérieur) qui est explicitement (thématiquement) au centre des écrits de Beckett, Tsevestaia ou Joyce / et de ma propre interprétation du «sur-réalisme».

FB - Quelle est ton interprétation personnelle du surréalisme?

SD - Le Surréalisme m’a offert une certaine liberté. J’ai toujours «vu» le surréalisme comme un moyen de rassembler des contradictions "ordinaires» et, ce faisant, de les "expliciter» tout en exposant leur caractère contradictoire. Le surréalisme porte également sur des expériences «extrêmes» qui sont restituées à travers une forme de «norme». La «Pataphysique» d'Alfred Jarry - superposer un processus imaginaire à un processus réel (cette définition est par trop simplifiée) - est une référence constante dans mon travail. L'idée de Jarry, bien entendu, constitue une référence incontournable dans la pensée surréaliste. Artaud, Bataille, Duchamp, Bellmer, Aragon, Man Ray, etc. - cette «école» de pensée et ces procédés artistiques correspondent à un mode de ré-vision et de re-conceptualisation sans attaches. Ce régime de pensée m’a permis d'articuler mes propres «idées» qui modifient à vue les «règles de l'engagement» ou qui retirent aux «règles» leur fondement. Ce qui m'a permis de jouer sur l'absurdité. La vie est pour moi absurde - et les 


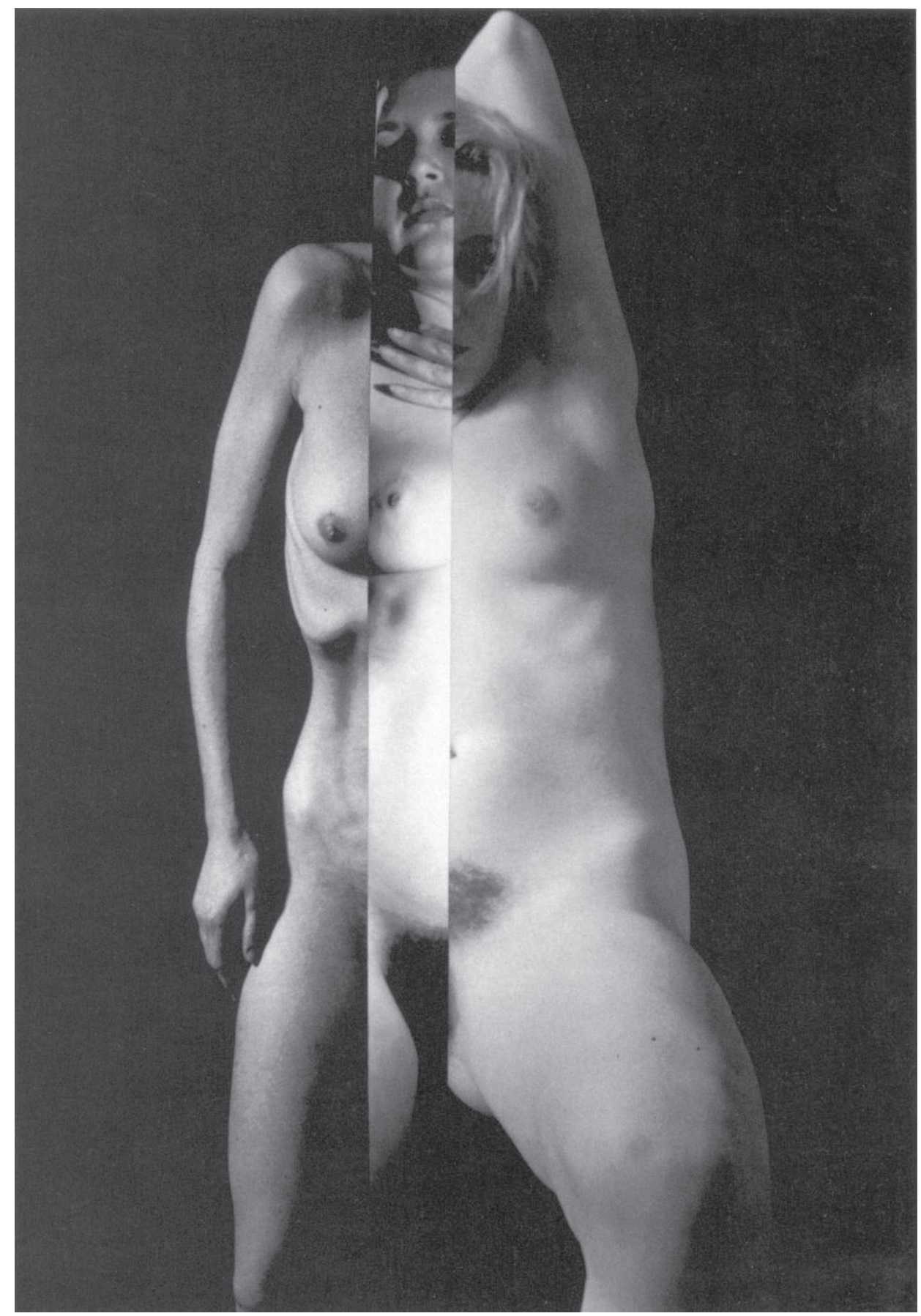


procédés surréalistes attribuent un sens à cette absurdité. Les procédés surréalistes restituent (ou attribuent) une signification profonde à cette absurdité, en affirmant: «il s'agit là de la réalité». Enfin, la réalisation d’un film répond également à un procédé surréaliste.

FB - Lorsque tu fais référence à un "assemblage" de choses qui constituent normalement des "contradictions", penses-tu à une forme de collage ou de montage? Comment peut-on relier ce procédé à tes propres photomontages, $\mathrm{Ha}$ Ha! ou la solution imaginaire (1993)? Enfin, y at-il une relation directe entre ton interprétation personnelle du surréalisme et $\boldsymbol{\nabla}$ Further and Particular (1988)?

SD - Le collage ou le montage (ou le découpage) constitue, en effet, un «assemblage» et, de façon plus générale, un procédé «surréaliste». Quels que soient les éléments "assemblés» (ou rapprochés), ceux-ci finissent par constituer un "énoncé» ou à générer des relations. De la sorte, on peut assembler deux images apparemment divergentes et ainsi les faire entrer en une relation réciproque. (Tu peux assembler deux couleurs divergentes qui alors forment tout aussi bien une relation unique!) Ces rapprochements générent une nouvelle signification au-delà de ce que chaque élément individuel représente en lui-même. Un élément peut entrer en contradiction avec un autre, mais ils ne représentent plus une contradiction dès lors qu'ils sont assemblés. Une nouvelle "logique» (une nouvelle narration) se forme. Ce procédé est fondamental dans $\mathrm{Ha}, \mathrm{Ha}$ ! En ce qui concerne $>$ Further and Particular - oui, il s'agit d'une application directe du surréalisme puisque le film cherche à conjoindre des idées de Jarry et de Bataille. Je pense que l'application est beaucoup trop littérale. Je préfère la façon dont j’ai utilisé des notions surréa-

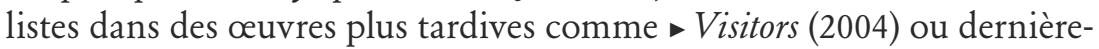
ment $\triangleright$ Oblivion (2005), où le sens du surréalisme est réarticulé à travers la réalisation et non pas appliqué littéralement.

\footnotetext{
FB - Further and Particular, tout comme $>0$ textes littéraires. Comment envisages-tu ton mode de transposition? De plus, les textes que tu transposes s'inscrivent dans une branche déviante du surréalisme (Jarry apparaît comme un modèle pour les surréalistes, Bataille suit sa propre ligne et fonde un groupe, tandis qu'Aragon représente l'aile politique du mouvement). Quelle relation entretiens-tu à ces textes?
}

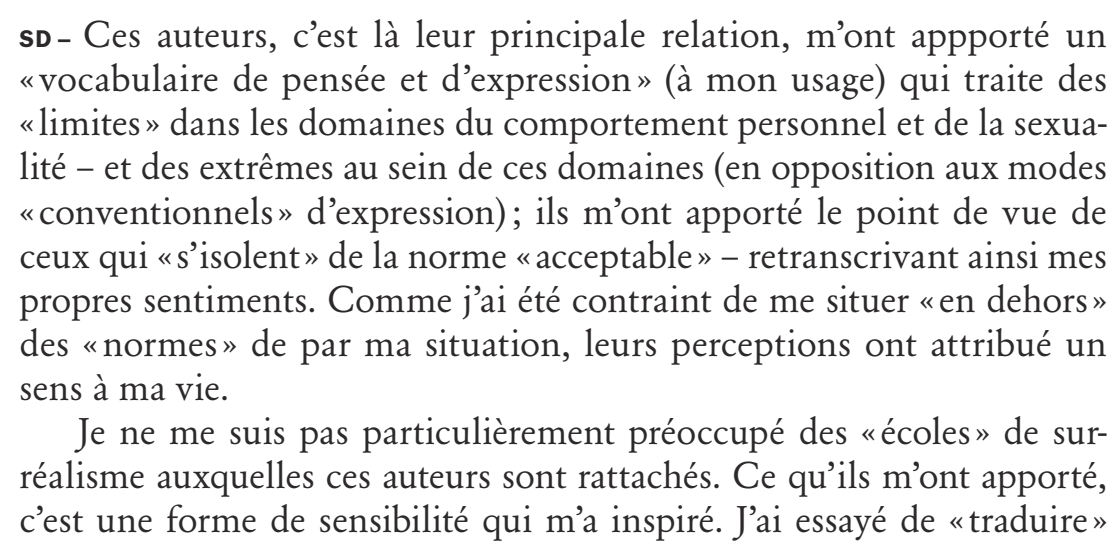




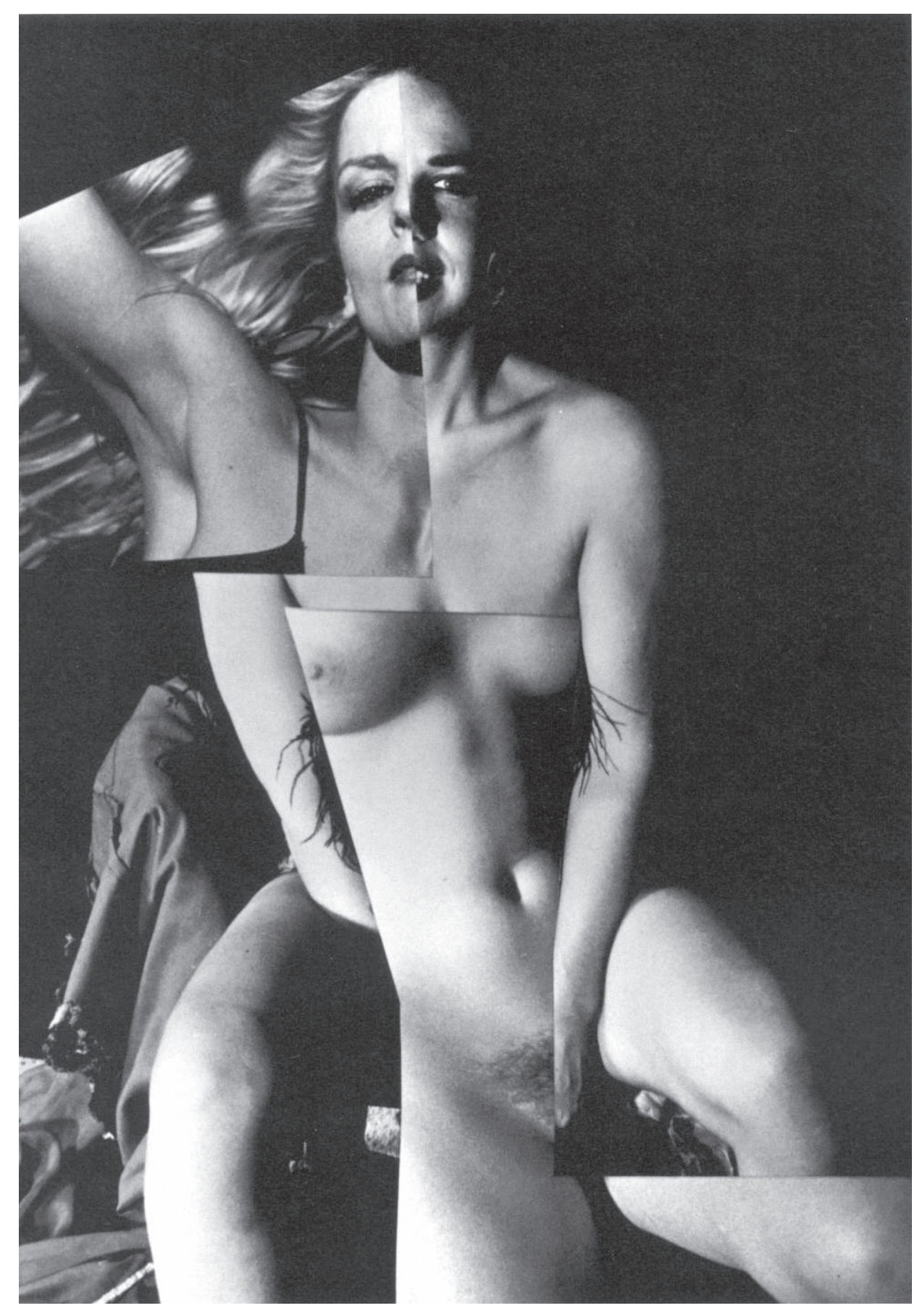


ou d'«exploiter» ce vocabulaire à travers mes préoccupations visuelles. Il s'agit de représenter à travers une forme visuelle les «sentiments» et les "sous-conversations» qu'ils mettent en jeu sur un plan littéraire, de les traduire à travers ma propre conversation, pour ainsi dire! D'habitude je n'essaye pas d'"adapter» leur narration $(\bullet$ Further and Particular est proche de cette démarche) mais de traduire ce mode de pensée à travers mon «histoire» personnelle (c'est de moi dont il est question le plus souvent - comme dans > Oblivion. Il s'agit d'une forme de "parenté»!) Je dois préciser - et c'est un point important - que ce ne sont pas seulement les surréalistes mais aussi des écrivains tels que Beckett (pour $・$ Another Time, 2002) et Joyce ( $\triangleright$ Times for, $\triangleright$ Soliloquy) qui m’ont inspiré, car - je ne sais pour quelle raison - ils s'efforcent tous d'exprimer l'espace intérieur du moi. C'est la principale impulsion de mon travail. Je ne suis pas un "narrateur». Je suis un "réflecteur» de cet espace intérieur. Ces auteurs m'ont aidé à trouver une "forme" pour exprimer en images ce qu'ils transmettent si bien à travers des mots. Pour répondre plus frontalement à ta question: c'est leur "poétique» que je m'efforce d'adapter (ou de métaphoriser) et non pas leurs histoires; et je le fais moins consciemment et littéralement qu'il ne peut y paraître.

\begin{abstract}
FB - Ce qui me frappe, c'est ta recherche cons trant sur des détails (i. e. gros plans, décadrage, etc.). Tu guettes ce qui se passe derrière les faits, en quelque sorte. Tu as rapporté l'intériorité à l'altérité, en parlant d'une frontière entre le comportement individuel (sexuel) et la normalité externe (sociétale). Peux-tu préciser par quels moyens tu réfléchis un espace intérieur, le tien aussi bien que celui des gens qui dévisagent la caméra? Et comment te situes-tu par rapport à la question des limites et de leur transgression, qui est centrale chez Artaud et Bataille?

Je me trompe peut-être. Mais je ne peux pas m'empêcher de relier l'écriture d'un flux de conscience (notoirement dans Ulysse mais en un sens aussi chez Beckett) à la notion d'un discours intérieur qui double (supplée) le film. Que penses-tu de cette relation?
\end{abstract}

SD - Je fais du cinéma pour me libérer et pour offrir au public des films tels qu'ils devraient être, plutôt que tels qu'ils sont. Mon but est aussi de réaliser des films qui explorent et qui expriment le moi. Exprimer le moi c'est aussi l'exposer, et dans le même temps ouvrir un dialogue avec les autres. Ce n'est pas seulement un dialogue, mais encore un processus d'investigation et de réflexion ouvert à tous. Pour pratiquer ce cinéma il faut (selon moi) être honnête et révélateur. Le spectateur doit pouvoir s'engager avec son identité et avec ses sentiments. Les films doivent donc s'ouvrir à un espace évasif et intime pour que le spectateur puisse y pénétrer et nourrir sa réflexion. Il peut s'agir d'un espace où le spectateur, comme le réalisateur, introduit sa propre forme de "narration", une «narration» qui n'est pas forcément définitive, ni close; ce processus n'intervient pas comme une histoire distrayante. Aussi pour moi, les films sont un miroir, comme c'est le cas dans mon cinéma. Leur réalisation suit un processus réflexif (souvent méditatif) qui agit dans le même temps comme un réflecteur. Ce qui implique un mode (ou un style) de réalisation qui se situe en-dehors ou au-delà des barrières de la «narration" conventionnelle (et au-delà même du "voyeurisme»). $\mathrm{Ce}$ que ressent le spectateur vis-à-vis de la mise en scène se produit 
également (dans la direction opposée) pendant le tournage entre l'«interprète", moi-même et la caméra!

Je pense que l'idée de "limites» et de leur «transgression" est imputable uniquement à Bataille ou Artaud, encore que leur prise de position est très précise sur la question. Dépasser les «limites» sexuelles atteint encore un degré supplémentaire avec Sade, bien sûr, mais je pense que la plupart des artistes sincères cherchent à dépasser les limites si l'on admet que l'idée de «limites» renvoie aux rituels oppressifs de maintien des conventions; ou à nos "limites" personnelles dues à nos "incapacités» et au désir de les élargir (ou de les explorer; ou de les confronter!). Pour moi, me confronter et m'efforcer de «dépasser» les "limites» constitue un moyen de prendre position sur (ou de me dresser contre) la solennité des rituels formels de la "convention" (de l'enfermement), un moyen de penser / de voir / de dépasser ce type d'"oppression".

Je pense que ta mise en relation est pertinente : la voix intérieure (le discours intérieur) contribue sans aucun doute au film.

FB - Y a-t-il une relation entre tes films et l'écriture d'un courant de conscience, telle que la pratique Gertrude Stein? Brakhage, par exemple, SD - A mon sens, c'est le soliloque de Joyce qui constitue le point de jonction le plus proche entre mon cinéma et un "courant de conscience» promouvait son œuvre comme un modèle d'écriture cinématographique. dans l'écriture. C'est plutôt un processus d'«associations» ou la façon dont les «conversations» ont cours qui est en jeu. Je ne me suis jamais vraiment attelé à l'écriture de Gertrude Stein, je ne peux donc pas me prononcer sur son style d'écriture.

FB - Behindert est l'un des premiers films où tu apparais face à la caméra. Comment as-tu appréhendé la question de l'autoreprésentation SD - Quand je me suis mis en scène dans les films, l'expérience s'apparentait à un point de pivot entre «être la caméra et puis montrer la caméra». et sa relation à un autre privilégié?

Dans $\neg$ Bebindert particulièrement (je le faisais pour la première fois), il s'agissait aussi de prendre part à un "dialogue" puisque Carola Regnier a filmé de son point de vue $90 \%$ des plans qui me représentent (sur un mode comparable au «home movie» avec une équipe, etc.). Je pense que cette relation se complexifie dans les films qui suivent - comme s'il s'agissait d'introduire une forme d'«autoportrait» et d'auto-réflection!

FB - Behindert met en jeu la question du portrait et de la rencontre plutôt que celle de l'auto-réflecSD - Behindert reposait, en majeure partie, sur une relation "réelle». Le tion. Comment as-tu approché Carola Regnier? Au début du film, elle semble presque extérieure à la début se présentait comme une «recréation" de notre rencontre (de notre conversation, isolée et faisant face à la caméra.. 
embarras) - comment nous nous sommes rencontrés! Le film ensuite se présente comme un "vivre ensemble» et sa forme s'apparente alors plus au "genre diaresque» (métaphoriquement, et non littéralement); il est question de «nous» et de notre «situation» (sa difficulté). Nos liens se rompent. Elle quitte l'« histoire».

FB - Tes films suivants sont $>$ Central Bazaar (1976) et $\downarrow$ The Silent Cry (1977). En un sens,

ils concluent une période (tu passes alors à l'autobiographie et au documentaire, pour le dire rapidement). Dans le premier, tu auscultes une dynamique de groupe, et dans le second tu te concentres sur une famille. Peut-on dire qu'il y a un lien entre eux?

- Central Bazaar met en scène la question de la mascarade, du travestissement, qui n'est d'habitude pas aussi présente dans ton œuvre. Ce qui aurait pu constituer une fête joyeuse et transgressive s'avère être bien plus étrange et inquiétant. Peux-tu préciser quelles étaient les conditions de tournage et tes intentions à l'époque?
SD - Il y a un lien, en général, entre $\bullet$ Central Bazaar et $\bullet$ The Silent Cry en ce sens que tous deux traitent du désir et de l'incapacité à satisfaire ce désir. Selon moi, ce lien réapparaît, à divers degrés, dans la plupart de mes films. Central Bazaar a plus de traits en commun avec un film comme -DynAmo - avec le "masque» (la Mascarade comme un faux spectacle simulé ; comme une feintise) et le "démasquage» du véritable sentiment. - Central Bazaar débusque la futilité derrière le masque, l'impossibilité à réaliser ses "fantasmes", ce qui provoque une forme de désespoir (ou encore quelque chose de plus étrange et inquiétant comme tu le fais remarquer). - The Silent Cry stigmatise la feintise "glaciale» attachée à la fausse proximité (la mascarade "sociale») qui est si commune dans les familles de classe moyenne, particulièrement anglaises, et ses effets sur une femme. Le film expose l'«auto-confusion» que celle-ci engendre; les "crises» d'identité ou la forme d'impuissance personnelle qu'elle génère. (L'illusion que quelque chose peut encore se produire (le désir) mais le refus de reconnaître que le désir se passe de toute compréhension. Cette toile de fond est à l'origine de l'«auto-déperdition».) [ $\checkmark$ The Silent Cry devait à l'origine constituer une trilogie avec $\triangleright$ Bebindert et $\triangleright$ Outside In (1981) pour la ZDF qui portait sur différentes formes de «handicaps»!]

- Central Bazaar a été tourné avant $>$ Behindert mais terminé après. J'ai réalisé - Central Bazaar avec l'accord des participants qui devaient tous (à l'exception d'une personne) vivre un mois ensemble. L'idée, comme tu l'as observé, était qu'ils participent tous à un repas de fête et qu'ils mettent par la suite en scène leurs "fantasmes" avec un ou plusieurs invités, et avec la caméra. (Il s'agit en quelque sorte d'une "rencontre de groupe».) Cette situation produit un large spectre de "frustrations", d'«évitements", d'aggressions, de désespoirs, de peurs décliné durant le tournage. Une personne (un hôte étranger joué par Carola Regnier) interrompt le groupe à trois reprises - c'est un hôte privilégié - pour contre-balancer la «solidité» du groupe. Le titre •Central Bazaar renvoie au nom d'un magasin où tu peux acheter tout ce que tu veux - ou qui semble posséder tout ce que tu peux bien vouloir acheter - si bien qu'en fin de compte tu n'achètes rien. C'est une métaphore du film, et 
de mes intentions. Lorsque nous avons accès à tout ce que nous désirons, nous ne sommes pas en mesure de dépasser nos limitations - ou si nous sommes en mesure de satisfaire nos désirs satisfaits, nous n'en éprouvons plus.

FB - Comment conçois-tu le lien entre deux films qui forment de mon point de vue un tout autobiographique, $>$ Outside In et $>$ Trying to Kiss the Moon (1994)? Selon quelles modalités les points de vue internes et externes s'inversentils dans le premier film? De plus, peut-on soutenir que Outside In entretient un lien avec le burlesque ou le genre comique, tout particulièrement en ce qui concerne la chute de ton SD - Il est vrai que $>$ Outside In tout comme $>$ Trying to Kiss the Moon sont autobiographiques en apparence. Cependant, $\rightarrow$ Trying to Kiss the Moon est plus autobiographique dans le sens «classique» du genre - il s'agit de réfléchir «toute» une vie (comme dans Mon Autobiographie de William Carlos Williams) à partir d'extraits d'images réelles de ma vie (par ex. : des home movies, des photos, des lettres, etc.) - et très peu de corps? «recréations».

- Outside In constitue un «terrain de jeu» très différent puisque le film repose exclusivement sur des recréations qui exposent l'absurdité, la stupidité même, des rencontres entre «une personne handicapée» et la société soi-disant "normale», avec son environnement. Comme la société «normale» (ou les individus en son sein) n'a aucun moyen d'entrer en relation avec le "handicap", cela produit un monde à la Marx Brothers - un burlesque, et même des types de "confusions» encore plus folles entre l'attendu et l'inattendu. La plupart des handicapés que je connais entrent facilement en rapport avec $>$ Outside In, parce qu'ils ont connu une «série d'histoires» similaires ou semblables à celles que j’ai vécues. Pour cette raison, le film est moins autobiographique : il a plus à voir avec ce qui se passe lorsqu'une personne handicapée entre en contact avec le monde "normal» quotidien. Le handicap met à mal toutes les normes attendues. Il met en évidence à quel point tout est vraiment absurde (Jarry aurait apprécié cela!). Outside In «embarrasse» souvent certaines personnes qui ne savent pas si elles peuvent rire face aux éléments «comiques» (par ex. la chute). Le film met aussi en «contraste» le monde «tranquille» et solitaire du handicapé (c'est-àdire moi) et le monde sens dessus-dessous qui survient lorsque tu quittes le monde "solitaire». Car avec un handicap (mon handicap) il n'y a pas de «moyen terme» - il n'y a pas de «normalité»!

FB - Trying to Kiss the Moon expose un point de vue kaléidoscopique. Comment avais-tu prévu SD - Avec $>$ Trying to Kiss the Moon j'assemblais pour la première fois de traiter le matériau hétérogène auquel tu as presque littéralement (dans mon cas, il s'entend!) des matériaux filmiques comme dans une peinture (ou un collage). Le film a été réalisé sans recours? 
plan structurel préconçu. Métaphoriquement, c'était comme ouvrir un tiroir et y trouver épars tous les morceaux de sa propre vie, et ensuite les récolter et essayer de les raccomoder (comme dans l'art "trouvé»!). Ma tâche a été facilitée par le fait que mon père avait une vaste collection de vieux «home movies» de famille. Il avait fait et conservé des rouleaux et des rouleaux de pellicule $8 \mathrm{~mm}$ qu'il tournait depuis que j'étais né. Il était un avide "opérateur» de films de famille: il a documenté autant que possible tous les événements familiaux. Comme la plupart des films $8 \mathrm{~mm}$ ont été sérieusement endommagés (au point d'être parfois inutilisables) j'ai eu l'idée d'utiliser les «textures" provoquées par la détérioration. Ensuite, il y avait des lots de photographies qui, une fois filmées, produisaient d'autres textures encore; et enfin il y avait mes vieux films et mes vieilles vidéos, etc. - procurant tous une riche "tapisserie» de sensations et de sentiments. Tout cela a été assemblé sur un mode très proche d'un "courant de conscience» ou de mes propres divagations et associations mentales. Comme je l'ai déjà dit - il n'y avait pas de plan concret (excepté l'idée) lorsque j'ai commencé le film. Lorsqu'un fragment se présentait, il en suggérait un autre et l'opération se poursuivait ainsi jusqu'à la fin. Heureusement j'avais reçu un peu d'argent "expérimental» (une chose rare) qui m’a permis de convertir les différents formats dans le format final du film.

FB - Peut-on concevoir $>$ Pain Is (1997) et $\boldsymbol{\nabla}$ Face of our Fear (1992) comme un prolongement

des films autobiographiques précédents, mais en adoptant un autre point de vue (impliquant un aspect plus documentaire)?

SD - Je ne considère pas $\bullet$ Pain Is ou $\bullet$ Face of our Fear comme des films "autobiographiques", même si j'entretiens un intérêt personnel à leurs thèmes. Ils sont nettement plus universels et traitent de problèmes sociaux - qui sont très importants. Ils constituent plutôt des essais sur ces sujets - même si l'on peut dire qu'il s'agit là d'un point de vue personnel (tel qu'il s'oppose à un "point de vue scientifique et purement objectif»). - Face of our Fear est un essai qui porte sur ce qui constitue probablement le plus grand préjudice - contre le handicap et la difformité - qui est ancré dans toutes les cultures et bien plus profondément que le préjudice racial ou religieux - c'était mon message. Même si le film est "stylistiquement» comparable à d'autres travaux, le sujet est plus historique et analytique. > Pain Is est moins « historique», mais porte encore sur une "problématique» universelle et non reconnue (ou acceptée) par la société et la communauté scientifique, ni comprise par la majorité. Le film me semble constituer un examen important de notre façon de penser et de l'influence que la "culture» exerce sur notre façon de nous exprimer. (En fait, c'est bien plus compliqué que cela!) Il est intéressant 
de relever que parmi tous les pays européens, seule la Grande-Bretagne a refusé de montrer ce film (parmi sept autres pays qui l'ont également rejeté) sous prétexte qu'il n'était pas assez «scientifique»! La GrandeBretagne a également rejeté - Face of our Fear alors même que la TV anglaise avait financé sa production - mais la politique qui entoure mes films constitue une autre histoire! Pour répondre brièvement à ta question: je ne considère pas que ces deux films participent à l'ensemble de l'autobiographie filmique.

FB - Quelle était le fondement, le point de départ, de ta correspondance vidéo avec Robert Kramer ( Video Letters (with Robert Kramer), 1991-2000)? Et ta relation à sa propre pratique SD - Les lettres vidéo avec Robert Kramer ont débuté sans véritablement prendre en considération nos pratiques filmiques respectives - si ce n'est filmique? que nous pratiquions tous deux le cinéma depuis un point de vue très "personnel». Nous avons d'abords entrepris ces lettres du point de vue de deux amis et de deux Américains vivant en Europe (nous avons le même âge et nous avons grandi dans la même ville). Nous n’avions aucune idée de la forme que ces lettres allaient prendre en termes "filmiques", mais il s'agissait d'un mouvement de "retour aux sources» en termes techniques - et avec la spontanéité du nouveau support vidéo à cette époque. Nous avons commencé par un débat personnel (du moins de notre point de vue) qui consistait à nous demander où nous nous situions vis-à-vis de nous-mêmes (plutôt qu'en fonction de «notre travail»). La question, de nos points de vue respectifs, portait sur l'opposition entre l'Europe et l'Amérique et sur notre incapacité à déterminer à quelle "culture» nous appartenions: ne nous conformant ni à l'une ni à l'autre (et n'étant pas vraiment intégrés par les deux lieux!), devions-nous opérer un «retour» en Europe ou bien "rester» sur place? Non sans ironie, cette problématique est à peine effleurée dans les lettres vidéo; néanmoins, elle nous a incité à réaliser ces lettres. (Nous étions sur le point de poursuivre cette correspondance vidéo lorsque malheureusement et subitement Robert est mort!) Tourner ces lettres - pour tous deux - a exercé une certaine influence sur nos films (à la suite du premier échange épistolaire) en termes de leur "mode de construction" et de notre approche stylistique. (Il est difficile de déterminer la nature spécifique de cette approche - mais les lettres ont certainement influencé notre façon de manipuler et de concevoir les matériaux.) 
FB - Peux-tu me dire comment tu as approché et traité la représentation d'une activité artistique, dans des films aussi différents que - Shadows from Light (1983, sur un photographe, Bill Brandt), Ballet Black (1986, sur la danse et la chorégraphie) et $\boldsymbol{B}$ Brendan Behan (1990, sur un écrivain)?

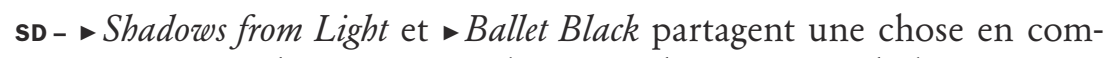
mun au niveau de mon approche. Tous deux partent de l'«intérieur» d'une personne, ou de gens impliqués. Je connaissais Bill Brandt (ou, plus précisément, j'étais un ami proche de son frère, Rolf, qui m'a appris à connaître Bill). Pour $\bullet$ Ballet Black je connaissais deux membres à l'origine de la compagnie de danse : ils m’ont donné un aperçu de leur travail et ont suscité mon intérêt pour ce qu'ils créaient à partir d'une certaine forme de danse. Bien entendu, la photographie et la danse m'ont toujours intéressé en tant que telles, mais l'opportunité unique de me situer à «l'intérieur» de leur "histoire créative» a constitué pour moi une importante source d'inspiration et de motivation. Dans $>$ Shadowes from Light, j'ai construit le film à partir de la perspective de Bill Brandt sur son œuvre. Il ne voulait jamais parler de son travail d'un point de vue technique. Lui-même était inspiré : il façonnait ses images en suivant une manière de penser très "rêveuse» et était ému par des travaux tels que Alice au pays des merveilles ou Kane et les Amberson de Welles. J'ai conçu la réalisation du film en "adoptant» ses références. De plus, Brandt m’a dit que les «nus» constituaient à ses yeux son travail le plus important, et partant le plus personnelle, car c'étaient les seules pièces qu'il avait réalisées en l'absence de toute "commission». Enfin, en connaissant «sa famille», j'ai commencé à comprendre ce qui était important pour Brandt, et ainsi à mieux comprendre son travail, à pouvoir mieux l'exprimer. Cela m'a aussi permis de me familiariser avec son monde que je pouvais plus facilement rattacher au mien (subjectivement), aussi l'ensemble du processus est devenu une "exploration" personnelle entre lui, moi et nos deux modes de travaux artistiques. (Ce processus "personnel» ou "subjectif» perturbe souvent les gens qui ne reherchent que le point de vue technique «objectif»). En un sens, cette relation "personnelle» a encore impliqué Brandt dans "la réalisation du film», celui-ci ne constituant plus seulement le sujet du film. De façon analogue, avec $\bullet$ Ballet Black, l'engagement personnel avec les amis qui constituaient à l'origine le «Ballet Nègre" m'a permis d'approcher de près ce qu'ils tentaient de faire sur un plan créatif, et dans leur cas, sur un plan politique également. Sans cette relation il aurait été virtuellement impossible de recréer le type de danses et de récits par la danse qui étaient au cœur de leur pensée. (La première fois qu'ils se sont produits en Angleterre, on qualifiait simplement leur spectacle de "danse simiesque " sans jamais prendre au sérieux leur tentative d'exprimer des «récits» ethniques en combinant les mouvements de la danse caribéenne et du ballet classique.) Quant à moi, personnellement, la manière dont d'autres «artistes", comme Brandt ou 
les membres du Ballet, trouvent leur motivation ou des moyens de manifester leur travail est aussi important que l'œuvre elle-même! (Ce qui les guide; ce qui les influence; comment ils ressentent leur propre vie et situation - et comment tout cela façonne leur œuvre!)

Ainsi, j'ai approché avec une attitude similaire $>$ Brendan Behan (même s'il y avait une "commission" alors que les deux autres films étaient mes propres propositions). L'écriture propre à Behan et la capacité qu'il avait à écrire comme il parlait ont déterminé mon approche dans le film. Il s'agissait de lui permettre de parler à travers des acteurs qui prononcent ses propres paroles qui se réfèrent à lui-même (comme s'il écrivait toujours ouvertement sa propre biographie en errant dans un bar ou le long du canal!)

Ces trois films portent sur trois activités «artistiques» différentes mais sur le même mode de processus. Les différences dépendent uniquement des manifestations personnelles qui conduisent à l'accomplissement de l'œuvre, c'est ce qui a déterminé la forme de mes films.

FB - Comment as-tu appréhendé $>$ Intoxicated by My IIIness (2001), qui tourne autour de la vieillesse, de la maladie et de l'attention portée SD - Intoxicated by My Illness représente une rupture et un déplacement dans ma vie, donc dans mon travail aussi, je suppose. Mon handicap à un patient? Ce film induit-il un point de rupture, de déplacement, dans ton travail? s'était dangereusement aggravé et je tombais malade très régulièrement, passant de plus en plus de temps à l'hôpital. Mon corps commença très visiblement à «tomber en morceaux», en partie à cause du «vieillissement» qui semblait précipiter d'autres problèmes physiques provoqués à l'origine par la polio, etc. Ma perte accrue de mobilité et de force a provoqué une rupture au sein de mes relations antérieures avec les gens, en particulier les relations personnelles, tout comme elle a bouleversé la façon dont j’avais été jusqu'alors capable de travailler. Mon état a d'autant plus limité ma capacité à travailler avec l'appareillage filmique (trop lourd; trop cher; etc.); aussi je me suis tourné vers le support digital (plus léger, meilleur marché et ajusté à un espace plus confiné). C'était aussi une époque où tous les "fonds» et les supports financiers faisaient défaut. Toutes mes demandes d'aide à la production étaient rejetées (elles le sont encore). Intoxicated a pris forme lorsque j'étais malade à l'hôpital et que je lisais un livre d'Anatole Broyard intitulé Intoxicated by My Illness, un écrivain américain atteint d'un cancer au stade terminal. (Je l'ai découvert à la suite de mes recherches pour le film $\bullet$ Pain!) Son «essai» m’a poussé à "considérer la maladie (ma propre maladie ) depuis l'intérieur»; à exprimer ce que je ressentais vis-à-vis de la situation dans laquelle je me trouvais: une grave maladie et la confrontation au vieillissement (le 
vieillissement en tant que processus terminal), les peurs et les désirs qui sont prisonniers d'un tel "espace» - comment on ressent cette situation et comment les autres y répondent (ou n’y répondent pas)!

Broyard disait que dans des situations aussi extrêmes que la maladie et la mort on est contraint à "parler» et à utiliser le «langage» d'autrui pour maintenir une conversation ; s'il ne s'y conforme pas, un patient qui garde le silence dans la maladie est réduit à "néant». Il faut considérer la maladie, disait-il, comme un voyage dans une contrée inconnue dont vous ignorez tout; comme un discours que vous tenez sur un sujet que vous ne maîtrisez pas; ou comme faire l'amour avec une femme démente!

- Intoxicated by My Illness (évidemment, le titre a été emprunté à Broyard) comporte deux parties, car pendant le tournage je suis devenu malade au point de passer de vie à trépas et de ne pas parvenir à terminer le film! Ainsi, la seconde partie exprime très clairement cette (ou mon) expérience de la mort cérébrale. Cela s’apparentait (en réalité) à un film, à un rêve étrange ou à un cirque bizarre où les pensées sexuelles se mêlent étroitement à des intrusions médicales indifférentes, l'ensemble de la scène acquérant le statut d'un événement théâtral. C'est tout aussi bien un espace de la perte, de la solitude et de la dépendance qu'un "drame intensifié» (d'où la présence de la musique) - le monde ultime de la contradiction. Sans aucun doute, Intoxicated by My Illness constitue donc un point de pivot dans mon œuvre qui traite dès lors d'un sens (ou d'une absence de sens) de la perte, de la vieillesse, de la mémoire, de la dépendance et de la mort - éprouvées dans la sphère intime du sujet. Sur un plan technique également, le film constitue un point de pivot: je suis parvenu à appliquer une pensée filmique au support digital, à explorer le potentiel expressif des procédés digitaux en vue de continuer à faire des «films» dans de nouvelles circonstances.

\footnotetext{
FB - Peux-tu me dire un mot sur ton travail après qui constituent en un sens des portraits filmiques, comme Some Friends (2002), $>$ Dad (2003), D Dear Frances (2003), \ Grandpère's Pear (2003). Un certain nombre d'entre eux s'apparentent à une évocation nostalgique, à une élégie, alors que d'autres exposent des instants de beauté, de bonheur et de partage. Peut-on dire qu'ils entretiennent des liens aux home movies? Tu t'es aussi tourné vers une forme alternative de fiction, comme dans $>$ Lost Dreams (2003) et $\boldsymbol{D}$ Another Time. Comment as-tu appréhendé la question d'une narration "indirecte" dans ces films?
}

-Intoxicated? Tu as réalisé une série de films

SD - Je suppose qu'on peut soutenir que la série de films comprenant - Some Friends, Dad, Dear Frances, etc. sont, en un sens, issus des aspects autoréflexifs de $\bullet$ Intoxicated. Bien qu'ils recourent à du matériau de "home movie» comme une "source», je ne les appellerais pas pour autant des «home movies». Ils sont bien plus "formels" (une formalisation des homes movies - en quelque sorte) et se rattachent plutôt à la notion de "portraits» (comme dans la peinture) ou de "poèmes» (de sonnets) (comme dans l'écriture) ou de "pièces de chambre» (comme dans la musique). Ils reposent sur des aspects réflexifs de "ma propre histoire» (la nostalgie, le souvenir, la commémoration, etc.). Ils tournent 
autour de la "perte», de l'"absence», et de la solitude. (Paul Willemen a dit qu'ils traitaient de la mort!) $\sim$ Friends apart porte à la fois sur la question de "ce que sont les amis"/où sont ceux que nous appelons des amis - des relations qui "autrefois étaient» et aujourd'hui ne sont plus! $>$ Dad et $\triangleright$ Dear Frances constituent littéralement des élégies - il est question de la perte d'êtres aimés $(\checkmark$ Dad étant plus réflexif puisque la perte remontait à des années déjà ; - Dear Frances constituant plutôt un panégyrique en réaction à une perte soudaine!). Lorsque je pense à ta question concernant mon "approche", une seule remarque me semble s'imposer: il s'agissait (il s'agit) en quelque sorte d'écrire des poèmes sur un plan visuel. Dans tous ces films, il s'agit d'un processus similaire de mon point de vue. Parfois cela me rappelle la façon dont Charles Ives tresse des élans musicaux dans ses pièces (pas seulement Ives, il s'entend!) (La musique dans $\bullet$ Dad est d'Ives.) Pour moi, $\bullet$ Dreams s'apparente aux autres formes brèves - il s'agit d'une réflexion sur la perte et l'absence. Cependant, avec $\bullet$ Another Time je me suis engagé dans une entreprise de reconstitution de ma propre situation depuis (ou à partir d') un cadre littéraire : je transpose en une expression visuelle la capacité qu'ont certains écrivains à articuler les mots en un mode de "courant de conscience». Dans • Another Time, il s'agit bien entendu de Malone meurt de Beckett. Ainsi, j'atteins à une narration «indirecte» puisque ce sont les "pensées» sans cesse changeantes, mouvantes, passées, actuelles des personnages qui s'imposent plutôt que les «aspects physiques du récit».

FB - Tu convoques Malone meurt comme une inspiration pour $\$ Another Time. Peux-tu préciser SD - Pour être plus précis, quant à Malone meurt, j'ai trouvé dans l'écriture de Beckett un moyen d'exprimer l'«espace intérieur» du "personnage» principal (qui incarne aussi d'une certaine façon la voix de l'auteur): l'écriture se meut à travers les pensées et combine le passé et le présent, la mémoire et l'imagination, l'événement et l'autoréflexion, sans jamais s'interrompre. L'inspiration (plutôt que l'influence) repose sur cette manière d'écrire, sur ce flux entre différents niveaux de "déclics» objectifs et subjectifs qui s'apparente à mes propres pensées. Le livre (et son mode d'écriture) est devenu un «guide» (ou une forme) pour façonner des éléments visuels suivant un mode comparable de dérive "incessante», qui permet de rapporter l'«histoire» (mon histoire) tout comme Beckett l'a racontée. Je suppose que l'on peut dire que j'ai suivi le «style» (ou la «manière») dont l'écriture exprime l'«histoire» plutôt que l'histoire elle-même (ou les personnages). Autrement dit, j'utilise «mon histoire» (et je suis le personnage) plutôt que celle de Beckett ou la façon dont tu as utilisé Beckett? La relation avec le texte semble plus distante que dans - Oblivion (où nous voyons le modèle lire Le Con d'Irène - dans $>$ Another Time nous voyons un livre de poésie mais pas Malone meurt). Pourtant, il y a des similitudes dans le traitement: la découpe de l'espace et des corps, les décadrages et le recours au ralenti, l'apparition d'ombres et la concentration sur des détails (ici un insecte, là une ampoule). Le son, dépourvu de dialogues, est discontinu, fragmentaire. Peux-tu m'en dire un peu plus sur les choix esthétiques que tu privilégies? 
de Malone. Je fais rarement référence au texte écrit et je n'utilise aucune des descriptions contenues dans le texte pour réaliser le film. Je n'ai recours qu'à ma seule "sensation» (ma sensation du texte) et je la mets en application pour réaliser le film. J'ai fait, plus ou moins, la même chose avec Le Con d'Irène (même si j'ai utilisé le même nombre de personnages qu'Aragon) mais j'ai suivi ma propre «histoire» (j'emploie le terme «histoire» très approximativement ici!) Quand j’ai réalisé • Another Time, certaines personnes m'ont dit que j'aurais dû mentionner Beckett et Malone meurt - le public aurait alors pu comprendre plus facilement le film. Aussi, quand j'ai réalisé -Oblivion j'ai inclu la référence visuelle au livre d'Aragon (comme un «truc» à la Hitchcock dans le film - n'importe quel livre aurait fait l'affaire) et j'ai crédité le livre au générique. Bien entendu, cela engendre un autre problème puisque le spectateur tente d'identifier les références au livre, ce qui peut distraire son attention - la plupart des relations «littérales» font défaut! (En Angleterre ce n'est pas vraiment un problème puisque presque personne ne connaît Aragon. Evidemment, ils connaissent Beckett!)

Les choix esthétiques sont très personnels puisqu'ils prennent place dans mon propre espace, mais la plupart de ces procédés reposent sur la clôture de l'espace, la perte de la "topographie» des lieux, la concentration sur les détails, ce qui survient "à l'extérieur", l'accroissement d'un détail ou d'un mouvement (ou la floculation du temps réel) - d'une certaine façon il s'agit de re-dramatiser un geste, un coup d'œil, une seconde (qui devient une minute), etc. Il s'agit d'égarements comme lorsque l'esprit divague: la focalisation varie comme une pensée qui tombe soudainement dans l'oubli. Le personnage (la caméra) s'enlise comme lorsqu'on évolue dans un rêve - ou lorsqu'on essaie de contrôler un rêve incontrôlable. Le son répond aux mêmes procédés. C'est une atmosphère angoissante de "bruits de la maison", du bruit du corps, de sons dans l'espace, de sons venant d'ailleurs, de bruits de pas, de portes qui se ferment, de chuchotements. C'est ce à quoi s'apparente être "coincé dans la solitude»! Tout paraît fragmentaire (comme si l'esprit se brisait - éparpillant des fragments - tout en se répétant continûment).

FB - Face Anthea (1990) constitue un portrait filmique, concentré sur une personne, un visage.

Quelle était ton intention en tournant cette longue séquence? Y a-t-il de ton point de vue une relation entre $>$ Face Anthea et des portraits plus tardifs comme, disons, $>$ Lost ou - Visitors?

SD $\rightarrow$ Face Anthea entretient beaucoup de liens avec des films plus anciens, comme $\triangleright$ Girl (1975), une partie de $\bullet$ DynAmo, peut-être $•$ Jesus' Blood. Il ne s'agit pas tant d'un portrait que d'un schéma mouvant d'«esprit» / de «pensée» tel qu'il est reflété par le visage (comme un miroir) - bien qu'il s'agisse aussi, de toute évidence, d'un portrait étiré dans le temps - 
mais qui n'a d'autre lieu que ce temps; il s'agit de cette heure seulement, d'aucune autre heure. $(\sim$ Face Anthea est devenu un projet lorsque la vidéo $\mathrm{Hi}-8$ est apparue, autorisant soudainement une prise d'une heure sans coupure!)

FB - Tes derniers films sont tournés en vidéo (je suppose qu'il s'agit avant tout d'un choix SD - Assurément, l'utilisation de la vidéo (digitale) constitue un choix "économique» et pratique. Cependant, chaque type de support est porteur de ses propres qualités esthétiques et expressives qui peuvent être pratique et économique). Ce choix exerce-t-il une influence sur ton approche esthétique, sur ton travail formel et sur ton point de vue énonciatif? "exploitées " et instrumentalisées jusqu'à prendre part à la structure d'ensemble de l'œuvre. Avant la généralisation de la couleur on faisait des films en noir et blanc et on utilisait le noir et blanc comme un facteur qui participait à l'«expression". Par la suite, avec le film en couleur, on a utilisé la couleur. Aujourd'hui la vidéo digitale s'impose; celle-ci possède également ses propriétés intrinsèques qui peuvent être exploitées. Evidemment, je préférerais que mes films «vidéo» soient transposés sur support pellicule (c'est très coûteux!) mais avant tout pour pouvoir les "projeter» sur un écran large. Je pense toujours que la lumière projetée est plus «magique» (puissante) que l'image "reçue» (comme sur un écran télévisé). (Mais bientôt il n'y aura plus de caméras pour support pellicule, comme cela s'est déjà produit pour la photographie, le choix ne se posera donc même plus!)

Traduit par François Bovier (version anglaise disponible sur le site de la revue).

Filmographie (vérifiée par Stephen Dwoskin)

- Asleep (Etats-Unis, 1961, 4', 16mm)

- American Dream (E.-U., 1961, 3', 16mm)

- Alone (E.-U., 1963, 13', 16mm)

- Naissant (E.-U., 1964, 14', 16mm)

- Chinese Checkers (E.-U., 1963 / version sonore : Grande-Bretagne, 1965, 13', 16mm)

- Soliloquy (E.-U., 1964 / version sonore: G.-B., 1967, 8 ', 16mm)

- Me, Myself and I (G.-B., 1967, 18', 16mm)

- Take Me (G.-B., 1968, 30', 16mm)

-Moment (G.-B., 1968-1969, 13', 16mm)

- Trixi (G.-B., 1969, 30', 16mm)

- To Tea (G.-B., 1970, 30', 16mm)
-C-Film (G.-B., 1970, 30', 16mm)

- Dirty (G.-B., 1965-1971, 15', 16mm)

- Times for (G.-B., 1971, 80', 16mm)

- Jesus’ Blood (Never Failed Me Yet) (G.-B., 1972, $30 ', 16 \mathrm{~mm})$

-DynAmo (G.-B., 1972, 120', 16mm)

-Tod und Teufel (G.-B., 1973, 94', 16mm)

- Behindert (G.-B./ Allemagne, 1974, 96', 16mm)

- Laboured Party (G.-B., 1975, 20', 16mm)

-Just Waiting (All., 1975, 10', 16mm)

- Girl (G.-B., 1975, 30', 16mm)

- Central Bazaar (G.-B., 1976, 156', 16mm)

- Kleiner Vogel (All., 1976, 40', 16mm)

- The Silent Cry (G.-B. / All. / France, 1977, 96', 16mm) 
-Outside in (G.-B./ All., 1981, 105', 16mm)

- Shadowes from Light (G.-B., 1983, 60', 16mm)

- Ballet Black (G.-B., 1986, 83', 16mm)

- Further and Particular (G.-B. / Fr., 1988, 110', 16mm)

- The Spirit of Brendan Behan (G.-B. / Fr., 1990, $30 ', 16 \mathrm{~mm})$

- Face Anthea (G.-B., 1990, 61', vidéo)

- Face of our Fear (G.-B., 1992, 53', vidéo)

- Trying to Kiss the Moon (G.-B., 1994, 96', 16mm)

- Pain Is (All./ G.-B. / Fr., 1997, 80', 16mm)

- Video Letters (with Robert Kramer) (G.-B., 1991-2000, 150 ', vidéo)
-Intoxicated by My Illness (G.-B., 2001, 62', vidéo)

- Another Time (G.-B., 2002, 52', vidéo)

- Some friends (Apart) (G.-B., 2002, 25', vidéo)

- Dad (G.-B., 2003, 15', vidéo)

- Dear Frances (In Memoriam) (G.-B., 2003, 18', vidéo)

- Grandpère’s Pear (G.-B., 2003, 5', vidéo)

- Lost Dreams (G.-B., 2003, 20', vidéo)

- Visitors (G.-B., 2004, 28', vidéo)

- Oblivion (G.-B., 2005, 78', vidéo)

- Before the Beginning (Work in Progress) (Belgique / G.-B., 2006, 120') / coréalisé avec Boris Lehman

Les deux photomontages sont tirés de

(Ed. The Smith, Brooklyn, 1993)

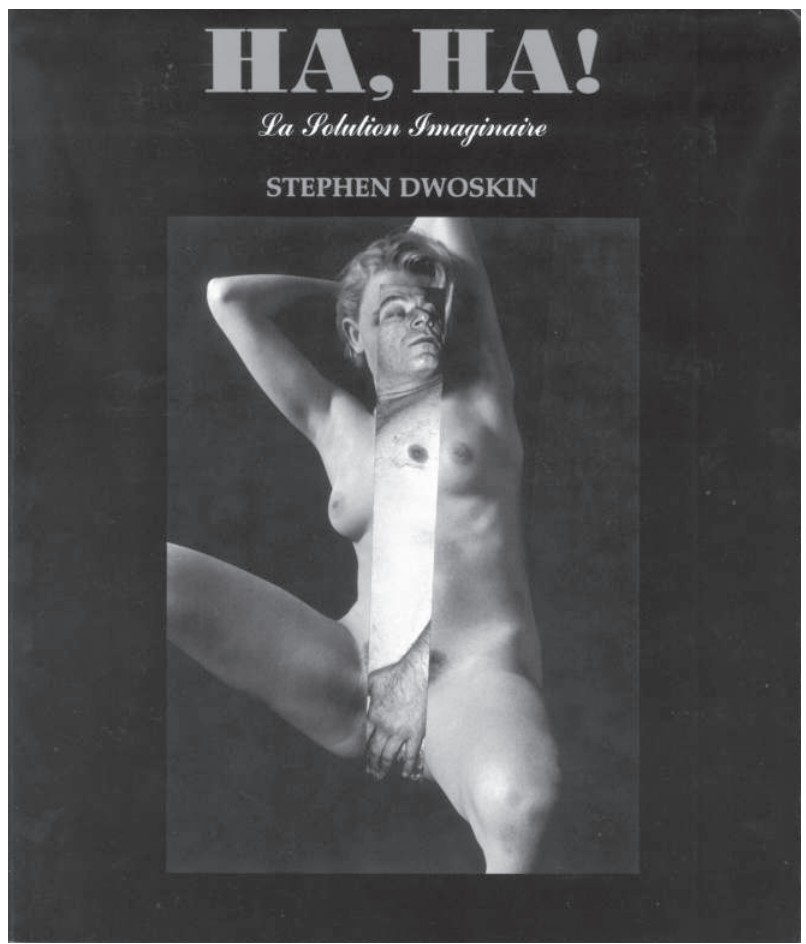

